\title{
Myelination of the Auditory Nerve: Functions and Pathology
}

\author{
Sergio Gonzalez-Gonzalez ${ }^{1 *}$ and Chantal Cazevieille ${ }^{2}$ \\ ${ }^{1}$ Boulevard de la Lironde, Parc Scientifique Agropolis, France \\ ${ }^{2}$ Institute for Neurosciences of Montpellier, Hospital Saint Eloi, France
}

*Corresponding author: Sergio Gonzalez-Gonzalez, CIL care, Boulevard de la

Lironde, Parc Scientifique Agropolis, Montpellier, France.

Received Date: February 01, 2019

Published Date: February 25, 2019

\begin{abstract}
Myelination is essential for the rapid propagation of action potentials along axons in both the central and peripheral nervous systems, and the Schwann cells are the responsible of myelin sheath production in the peripheral nervous system. In the cochlea, sensory hair cells and neurons are in close association with several types of glial cells. Whereas hair cells are surrounded by supporting cells, spiral ganglion neuron axons are myelinated by Schwann cells. This myelin contributes to axonal protection and allows for efficient action potential transmission along the auditory nerve. Because in the last decade, myelin research has been notably focusing on the molecular and cellular mechanism of demyelination process and the associated axonal loss, in this review we summarize the role of the myelin sheath in auditory nerve and how Schwann cell demyelination results in a reduction in the velocity of action potential propagation, and an increase in nerve conduction vulnerability.
\end{abstract}

Keywords: Myelin; Schwann cell; Auditory system; Hearing loss; Demyelination

\section{Schwann Cells and Myelin}

Myelination is essential for the rapid propagation of action potentials along axons in both the central (CNS) and peripheral (PNS) nervous systems. In the PNS, the myelin sheath is formed by Schwann cells (SCs) [1]. Myelinating Schwann cells wrap around axons so that the molecular machinery required to propagate action potentials is concentrated at regular sites, known as nodes of Ranvier. The scarcity of data regarding axon-glia physiology in peripheral nerves underscores the complexity of this cell-cell relationship and the relative inadequacy of the current approaches used in peripheral nerve research. Recent data have highlighted the role of subcellular organelles and molecular factors in the interaction between SC and axons [2]. This is consistent with the emerging opinion in the field that the interdependence between the axon and the myelinating glia is so deep that CNS and PNS neuropathies cannot be correctly addressed and treated without first understanding the extent and complexity of the relationship between neurons and glial cells [3]. The PNS shows a surprising capacity of regeneration compared to the CNS. This ability of peripheral nerves to recover quickly following damage is to a large extent due to the remarkable plasticity of Schwann cells [4].
SCs are derived from neural crest cells that differentiate into SC precursors and then into immature SCs between E12 and E15 in mice. Around birth, axonal sorting and myelination start in the peripheral nerves. Some SCs establish a 1:1 relationship with large-diameter axons, wrap them multiple times to form a thick and compact myelin sheath. Nerve homeostasis, trophic support and myelin maintenance are other important functions of SCs in adulthood [5]. The myelin geometry, diameter and internodal length, is therefore a critical parameter of the nerve conduction velocity and changes in this geometry underlie numerous functional properties of the brain and nerves [6].

Moreover, reduced internodal length, which strongly impairs nerve conduction velocity [6,7], is linked to human neuropathies such as multiple sclerosis in the central nervous system [8] and Charcot-Marie-Tooth (CMT) diseases [9-11] and congenital neuromuscular dystrophy $1 \mathrm{~A} \mathrm{[12]} \mathrm{in} \mathrm{the} \mathrm{peripheral} \mathrm{nervous} \mathrm{system.}$ While the molecular mechanisms responsible for the homogeneity of myelin thickness in Schwann cells along the same axon have been uncovered [13-15], the mechanisms responsible for myelin elongation and the formation of regular myelin lengths remain 
unknown. Besides their major roles in normal nerve physiology, SCs play a key function for repair in many pathological conditions thanks to their striking plasticity [16]. For example, after a peripheral nerve injury, they are capable of switching into a SC immature-like phenotype that drives nerve repair. Over the last decades, major progress has been made in unraveling molecular mechanisms and signaling pathways that drive SC dedifferentiation and regulate their plasticity. This high regenerative capacity opens the door to the development of new therapeutic approaches for the regeneration of peripheral axons after injury.

\section{Hearing Loss}

Hearing loss is the most common form of sensory impairment in humans, affecting 360 million persons worldwide, with a prevalence of 183 million adult males and 145 million adult females. In no syndromic deafness, only hearing function is noticeably altered, whereas syndromic deafness is accompanied by other physiological defects. Hearing loss can be caused by environmental factors, such as exposure to noise or ototoxic chemicals, or by aged related senescence. Traumatic injury, such as injury caused by exposure to an explosion or to the firing of a gun, can lead to sudden hearing loss. Sometimes this hearing loss is accompanied by the perception of a constant ringing noise called tinnitus [17]. Moreover, genetic factors as mutations in MT-TS1, MYO7A or ACTG1 genes [18,19], between many others, have already been linked to nonsyndromic hearing loss. Noise exposure is responsible for approximately $10 \%$ of hearing loss in adults, in particular military veterans [20]. Short impulses of high intensity noise such as a gunshot or explosion can trigger sudden hearing loss, which is generally irreversible and associated with structural tissue damage of cochlea and auditory nerve. Susceptibility to damaging effects of noise differs remarkably ampled to the ribbon synapses, whereas the myelinated axons extend from the habenula perforate to the SGN stomata. Once the central SGN process enters the CNS, it becomes myelinated by oligodendrocytes.

Two subpopulations of SGNs exist: type I, which account for 90\%-95\% of total SGNs, and type II, which comprise the remaining 5\%-10\% of SGNs. The cell bodies of both neuron types are housed within Rosenthal's canal and extend peripheral axons through the osseous spiral lamina to form connections with hair cells in the organ of Corte. Type I SGNs extend a single axon to a single inner hair cell (IHC) and type II SGNs innervate multiple outer hair cells (OHC) with numerous axonal projections [21]. Type I SGN somas are covered by myelinating satellite cells and their axons are unsheathed by myelinating Schwann cells. Conversely, type II SGNs are enveloped by non-myelinating satellite and Schwann cells. Recent studies have demonstrated that migration, maturity and survival of SGNs is largely dependent on surrounding glial cells $[22,23]$.

Additionally, conditional deletion of Sox10, a transcription factor regulating glial cell differentiation, results in reduced glial numbers, abnormal SGN distribution, decreased SGN migration, and the appearance of nerve fibers that overshoot the organ of Corte and extend into the cochlear lateral wall, revealing the important role glial cells play in proper SGN innervation [24].
However, whereas it is still unknown how glial cell numbers are regulated as the auditory nerve matures, the immune cells such as macrophages and microglia could contribute to nerve refinement in the central nervous system [25,26]. Finally, satellite glial cells express glutamine synthetase for converting ammonia and glutamate to glutamine [27], which is important for metabolizing excess glutamate and preventing excitotoxicity [28].

Satellite cells are also important for neuronal survival as knockout of sap B from these cells resulted in progressive neuronal death [29]. Complete regeneration of the spiral ganglion satellite cells leads to spontaneous proliferation and regeneration of satellite cells in other sensory ganglia [30-32]. Timely regeneration of the satellite cells and wrapping on SGN cell bodies may be important to preserve the survival and function of the SGNs.

\section{Demyelination and Hearing Loss}

As mentioned before, in the PNS, the Schwann cells are responsible for myelin production, which contributes to axonal protection and allows for efficient action potential transmission along the auditory nerve. Unfortunately, acquired and hereditary demyelinating diseases of the PNS are numerous and affect an increasing number of people [33]. Acquired demyelinating diseases are the most common, as they include diabetic peripheral neuropathy [34,35], drug-related peripheral neuropathies, leprosy, and inflammatory neuropathies [36]. Diabetic peripheral neuropathy is a major complication of diabetes type 1 and 2 and a cause of considerable morbidity $[37,38]$. Indeed, it has been reported that at least $50 \%$ of diabetic patients develop one or several forms of diabetic neuropathy within 25 years after diagnosis [37]. This neuropathy affects both myelinated SCs and peripheral axons/neurons, leading to changes in nerve conduction, and it is often associated with demyelination in the long term [39]. However, the molecular mechanisms that lead to these nerve defects remain unclear. In the PNS, hereditary demyelinating diseases are rare but remain among the most common hereditary diseases [40].

While they are rarely lethal, they range from life threatening to severely affecting life and therefore put a high burden on public health systems. The etiologies of acquired and hereditary peripheral nerve diseases are diverse, but they all result in demyelination and subsequent neuronal death. SCs themselves have the ability to turn against their own myelin and initiate myelin breakdown, in addition to being able to call on macrophages for myelin phagocytosis [41]. The maintenance of healthy myelin and normal nerve function depends on tight control of this intrinsic potential for myelin destruction. In contrast to SCs, the oligodendrocytes, the myelinforming cells of the CNS, appear to be unable to digest myelin, a feature that has been linked to poor regenerative ability of CNS tissue.

In spite of the central position of myelin breakdown in Schwann cell biology and pathology, the cellular and molecular mechanisms that make Schwann cell-mediated myelin digestion possible have not been established. However, some studies suggested phagocytosis as the mechanism by which SCs digest their myelin after nerve transection. In the first phase of myelin clearance, 
the SCs themselves break down $40-50 \%$ of the myelin during the first 5-7 d after injury [42]. Subsequently, macrophages that invade injured nerves play the major role in myelin breakdown by phagocytosis in conjunction with antibodies and complement. It is likely that SCs take part in phagocytosis of myelin debris during this second phase of myelin clearance $[43,44]$.

This SC demyelination results in an increase in membrane capacitance and a decrease in membrane resistance, leading to a delayed nerve excitation, a reduction in the velocity of action potential propagation, and an increase in nerve conduction vulnerability [45]. Fibers that are demyelinated to differing degrees conduct neural signals at different speeds, and the synchrony of discharges can be affected. Although neurons that are not entirely myelinated are capable of conducting action potentials, they do so with prolonged refractory periods and an impaired ability to transmit high-frequency pulse trains [45].

As a result, repetitive activation of demyelinated fibers results in a progressive increase in the conduction time of the action potential and may lead to an intermittent or total block in their propagation. The pathophysiologic changes in neural conduction properties associated with demyelination are likely to have profound effects on the auditory brainstem response which is reliant on the relatively precise synchronous response of a population of auditory nerve fibers to a transient acoustic stimulus. Moreover, in advanced demyelinated states, the propagation of the action potential becomes increasingly vulnerable, and the risk of depolarization block is increased, especially for the relatively repetitious stimuli observed in auditory nerve in order to generate the auditory brainstem response. The importance of auditory nerve demyelination is indicated by the occurrence of deafness in patients with CMT disease [46].

In rodent models, increased latency of wave I of the auditory brainstem response (ABR) has been linked to mutations affecting myelin [46]. Moreover, doxorubicin, a demyelinating agent, abolishes the ABR within $24 \mathrm{~h}$ of its injection within the auditory canal. Pilati and collaborators described loss of myelin from $50 \%$ of rat auditory nerve axons innervating basal cochlear inner hair cell synapses, 3-4 days after acoustic trauma [47]. Moreover, increasing number of studies demonstrated that acoustic trauma leading to hearing loss also triggered auditory nerve demyelination [48] and notable morphological changes at myelin sub-domains such as nodes of Ranvier, paranodes and juxtaparanodes that were associated with the decreased conduction velocity [49]. This acoustic trauma decreased the auditory nerve conduction velocity and the myelin thickness of the fibers projecting toward the auditory brainstem [49].

\section{Remyelinating Therapies}

In the last decade, myelin research has been notably focusing on the molecular and cellular mechanism of demyelination process and the associated axonal loss. To preserve axons from degeneration, the most promising strategy is to enhance remyelination of demyelinated axons. Remyelination can fail in patients because of SC recruitment, survival or differentiation, and some pro-remyelinating compounds are ongoing clinical trial [50].
Two types of therapeutic strategies related to myelination signals have been proposed. The first involves reducing the interference to myelination signals. Interference of such signals is posited to occur at all levels of signaling pathways, ranging from extracellular ligand-receptor interactions to transcription [51].

Genetic proof of principle experiments is underway in mouse models of hereditary neuropathies to limit expression of 'dedifferentiation' genes such as c-Jun or Sox2 in Schwann cells; no results have been published thus far. The second approach is to restore or augment normal myelination signaling during remyelination. The first preclinical and clinical trials along these lines have appeared in the last 6 years. For example, $70 \%$ of hereditary demyelinating neuropathies are caused by overexpression of peripheral myelin protein 22 in CMT disease type $1 \mathrm{~A}$. Therefore, reducing myelination signaling in order to normalize PMP22 expression might be of therapeutic benefit.

Preclinical trials of ascorbic acid, which might lower levels of cAMP [52] and the progesterone inhibitor onapristone [53] have demonstrated that, in principle, the reduction of PMP22 levels improves myelination in animal models of CMT1A. As a result, several clinical trials with ascorbic acid in patients with CMT1A are underway [54] and a search is ongoing for less toxic progesterone inhibitors than onapristone. Thyroid hormone T4 promotes oligodendrocyte differentiation and myelination in development and has been shown to promote myelin repair in a preclinical trial in acute experimental autoimmune encephalomyelitis models [55]. IGF-1 also augments myelination in the CNS and a phase II pilot study of the tolerability and efficacy of subcutaneously administered recombinant human IGF-1 (CEP-151) in patients with multiple sclerosis has been completed, although no results have been reported yet. In conclusion, the search for signaling molecules that promote remyelination holds promise for eventually developing combinatorial therapies, including existing anti-inflammatory interventions, for demyelinating diseases.

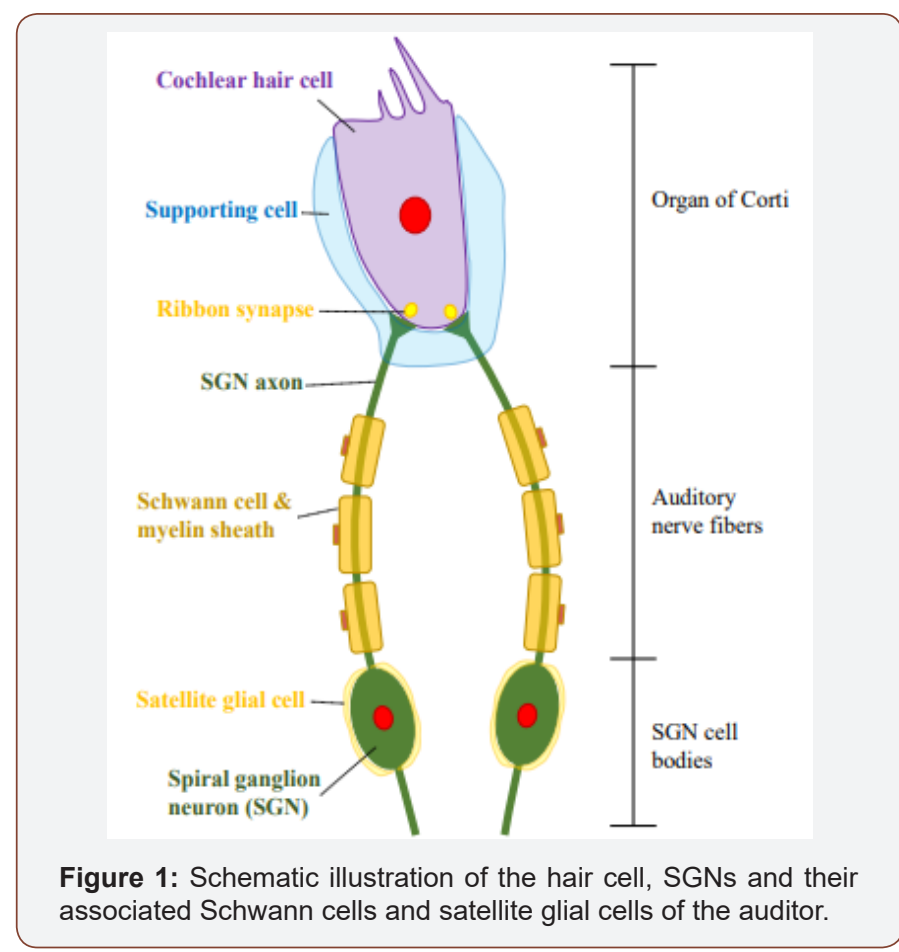


Future research into the development of such treatments will need to address two additional issues. First, for the various diseases of myelin, the initial site of damage (SC or axons) needs to be resolved, as this location might have consequences for where the drug is delivered. Indeed, the cell bodies of neurons and SC that form one myelin-axon unit sometimes reside in different parts of the nervous system, which are characterized by differing pharmacological barriers. Second, as evidence mounts for the important role of axonal injury in disability, signals originating in SC that mediate axonal support need to be identified and characterized (Figure 1).

\section{Acknowledgement}

We thank M Self for the accurate lecture of the manuscript.

\section{Conflict of Interest}

None.

\section{References}

1. Sherman DL, Brophy PJ (2005) Mechanisms of axon ensheathment and myelin growth. Nat Rev Neurosci 6(9): 683-690.

2. Viader A, Golden JP, Baloh RH, Schmidt RE, Hunter DA, et al. (2011) Schwann cell mitochondrial metabolism supports long-term axonal survival and peripheral nerve function. J Neurosci 31(28): 10128-10140.

3. Nave KA (2010) Myelination and support of axonal integrity by glia. Nature 468(7321): 244-252.

4. Jessen KR, Mirsky R, Lloyd AC (2015) Schwann Cells: Development and Role in Nerve Repair. Cold Spring Harb Perspect Biol 7(7): a020487.

5. Jessen KR, Mirsky R (2005) The origin and development of glial cells in peripheral nerves. Nat Rev Neurosci 6(9): 671-682.

6. Tomassy GS, Berger DR, Chen HH, Kasthuri N, Hayworth KJ, et al. (2014) Distinct profiles of myelin distribution along single axons of pyramidal neurons in the neocortex. Science 344(6181): 319-324.

7. Court FA, Sherman DL, Pratt T, Garry EM, Ribchester RR, et al. (2004) Restricted growth of Schwann cells lacking Cajal bands slows conduction in myelinated nerves. Nature 431(7005): 191-195.

8. Prineas JW, Connell F (1979) Remyelination in multiple sclerosis. Ann Neurol 5: 22-31.

9. Boerkoel CF, Takashima H, Stankiewicz P, Garcia CA, Leber SM, et al. (2001) Periaxin mutations cause recessive Dejerine-Sottas neuropathy. Am J Hum Genet 68: 325-333.

10. Gillespie CS, Sherman DL, Fleetwood-Walker SM, Cottrell DF, Tait S, et al. (2000) Peripheral demyelination and neuropathic pain behavior in periaxin-deficient mice. Neuron 26: 523-531.

11. Guilbot A, Williams A, Ravise N, Verny C, Brice A, et al. (2001) A mutation in periaxin is responsible for $\mathrm{CMT} 4 \mathrm{~F}$, an autosomal recessive form of Charcot-Marie-Tooth disease. Hum Mol Genet 10: 415-421.

12. Di Muzio A, De Angelis MV, Di Fulvio P, Ratti A, Pizzuti A, et al. (2003) Dysmyelinating sensory-motor neuropathy in merosindeficient congenital muscular dystrophy. Muscle Nerve 27: 500-506.

13. Cotter L, Ozcelik M, Jacob C, Pereira JA, Locher V, et al. (2010) Dlg1-PTEN interaction regulates myelin thickness to prevent damaging peripheral nerve overmyelination. Science 328: 1415-1418.

14. Michailov GV, Sereda MW, Brinkmann BG, Fischer TM, Haug B, et al. (2004) Axonal neuregulin-1 regulates myelin sheath thickness. Science 304: 700-703.

15. Taveggia C, Zanazzi G, Petrylak A, Yano H, Rosenbluth J, et al. (2005) Neuregulin-1 type III determines the ensheathment fate of axons. Neuron 47: 681-694.
16. Xu QG, Midha R, Martinez JA, Guo GF, Zochodne DW (2008) Facilitated sprouting in a peripheral nerve injury. Neuroscience 152(4): 877-887.

17. Knipper M, Zimmermann U, Muller M (2010) Molecular aspects of tinnitus. Hear Res 266(1-2): 60-69.

18. Tiranti V, Chariot P, Carella F, Toscano A, Soliveri P, et al. (1995) Maternally inherited hearing loss, ataxia and myoclonus associated with a novel point mutation in mitochondrial tRNASer (UCN) gene. Hum Mol Genet 4(8): 1421-1427.

19. Liu XZ, Walsh J, Mburu P, Kendrick-Jones J, Cope MJ, et al. (1997) Mutations in the myosin VIIA gene cause non-syndromic recessive deafness. Nat Genet 16(2): 188-190.

20. Gonzalez Gonzalez S (2018) Noise-Induced Hearing Loss and Tinnitus in Military Personnel. M J E Med 3(1): 27.

21. Marrs GS, Spirou GA (2012) Embryonic assembly of auditory circuits: spiral ganglion and brainstem. J Physiol 590(10): 2391-2408.

22. Hansen MR, Vijapurkar U, Koland JG, Green SH (2001) Reciprocal signaling between spiral ganglion neurons and Schwann cells involves neuregulin and neurotrophins. Hear Res 161(1-2): 87-98.

23. Jeon EJ, Xu N, Xu L, Hansen MR (2011) Influence of central glia on spiral ganglion neuron neurite growth. Neuroscience 177: 321-334.

24. Mao Y, Reiprich S, Wegner M, Fritzsch B (2014) Targeted deletion of Sox10 by Wnt1-cre defects neuronal migration and projection in the mouse inner ear. PLoS One 9(4): e94580.

25. Paolicelli RC, Bolasco G, Pagani F, Maggi L, Scianni M, etal. (2011) Synaptic pruning by microglia is necessary for normal brain development. Science 333(6048): 1456-1458.

26. Miron VE, Boyd A, Zhao JW, Yuen TJ, Ruckh JM, et al. (2013) M2 microglia and macrophages drive oligodendrocyte differentiation during CNS remyelination. Nat Neurosci 16(9): 1211-1218.

27. Eybalin M, Norenberg MD, Renard N (1996) Glutamine synthetase and glutamate metabolism in the guinea pig cochlea. Hear Res 101: 93-101.

28. Suarez I, Bodega G, Fernandez B (2002) Glutamine synthetase in brain: effect of ammonia. Neurochem Int 41: 123-142.

29. Akil O, Sun Y, Vijayakumar S, Zhang W, Ku T, et al. (2015) Spiral ganglion degeneration and hearing loss because of satellite cell death in saposin B-deficient mice. J Neurosci 35(7): 3263-3275.

30. Elson K, Simmons A, Speck P (2004) Satellite cell proliferation in murine sensory ganglia in response to scarification of the skin. Glia 45(1): 105109.

31. Nascimento DS, Castro-Lopes JM, Moreira Neto FL (2014) Satellite glial cells surrounding primary afferent neurons are activated and proliferate during monoarthritis in rats: is there a role for ATF3? PLoS ONE 9(9): e108152.

32. Donegan M, Kernisant M, Cua C, Jasmin L, Ohara P T (2013) Satellite glial cell proliferation in the trigeminal ganglia after chronic constriction injury of the infraorbital nerve. Glia 61(12): 2000-2008.

33. Hughes RA (2002) Peripheral neuropathy. BMJ 324(7335): 466-469.

34. Zochodne DW (2008) Diabetic polyneuropathy: an update. Curr Opin Neurol 21(5): 527-533.

35. Zenker J, Ziegler D, Chrast R (2013) Novel pathogenic pathways in diabetic neuropathy. Trends Neurosci 36(8): 439-449.

36. Pham K, Gupta R (2009) Understanding the mechanisms of entrapment neuropathies. Review article. Neurosurg Focus 26(2): E7.

37. Harati Y (1987) Diabetic peripheral neuropathies. Ann Intern Med 107(4): 546-559.

38. Sugimoto K, Murakawa Y, Sima AA (2000) Diabetic neuropathy- a continuing enigma. Diabetes Metab Rev 16(6): 408-433.

39. Malik RA (2014) Pathology of human diabetic neuropathy. Handb Clin Neurol 126: 249-259. 
40. Skre H (1974) Genetic and clinical aspects of Charcot-Marie-Tooth's disease. Clin Genet 6(2): 98-118.

41. Hirata K, Kawabuchi M (2002) Myelin phagocytosis by macrophages and nonmacrophages during Wallerian degeneration. Microsc Res Tech 57(6): 541-547.

42. Perry VH, Tsao JW, Fearn S, Brown MC (1995) Radiation-induced reductions in macrophage recruitment have only slight effects on myelin degeneration in sectioned peripheral nerves of mice. Eur J Neurosci $7(2): 271-280$.

43. Dubovy P, Jancalek R, Kubek T (2013) Role of inflammation and cytokines in peripheral nerve regeneration. Int Rev Neurobiol 108: 173-206.

44. Vargas ME, Watanabe J, Singh SJ, Robinson WH, Barres BA (2010) Endogenous antibodies promote rapid myelin clearance and effective axon regeneration after nerve injury. Proc Natl Acad Sci USA 107(26): 11993-11998.

45. Pender MP, Sears TA (1984) The pathophysiology of acute experimental allergic encephalomyelitis in the rabbit. Brain 107 (Pt 3): 699-726.

46. Starr A, Michalewski HJ, Zeng FG, Fujikawa-Brooks S, Linthicum F, et al. (2003) Pathology and physiology of auditory neuropathy with a novel mutation in the MPZ gene (Tyr145->Ser). Brain 126(Pt 7): 1604-1619.

47. Pilati N, Large C, Forsythe ID, Hamann M (2012) Acoustic over-exposure triggers burst firing in dorsal cochlear nucleus fusiform cells. Hear Res 283(1-2): 98-106.

48. Coyat C, Cazevieille C, Baudoux V, Larroze-Chicot P, Caumes B, et al. (2018) Morphological consequences of acoustic trauma on cochlear hair cells and the auditory nerve. Int J Neurosci 26: 1-16.
49. Tagoe T, Barker M, Jones A, Allcock N, Hamann M (2014) Auditory nerve perinodal dysmyelination in noise-induced hearing loss. J Neurosci 34(7): 2684-2688.

50. Plemel JR, Liu WQ, Yong VW (2017) Remyelination therapies: a new direction and challenge in multiple sclerosis. Nat Rev Drug Discov 16(9): 617-634.

51. Derfuss T, Parikh K, Velhin S, Braun M, Mathey E, et al. (2009) Contactin-2/ TAG-1-directed autoimmunity is identified in multiple sclerosis patients and mediates gray matter pathology in animals. Proc Natl Acad Sci USA 106(20): 8302-8307.

52. Passage E, Norreel JC, Noack-Fraissignes P, Sanguedolce V, Pizant J, et al. (2004) Ascorbic acid treatment corrects the phenotype of a mouse model of Charcot-Marie-Tooth disease. Nat Med 10(4): 396-401.

53. Sereda MW, Meyer zu Horste G, Suter U, Uzma N, Nave KA (2003) Therapeutic administration of progesterone antagonist in a model of Charcot-Marie-Tooth disease (CMT-1A). Nat Med 9(12): 1533-1537.

54. Burns J, Ouvrier RA, Yiu EM, Joseph PD, Kornberg AJ, et al. (2009) Ascorbic acid for Charcot-Marie-Tooth disease type 1A in children: a randomised, double-blind, placebo-controlled, safety and efficacy trial. Lancet Neurol 8(6): 537-544.

55. Fernandez M, Giuliani A, Pirondi S, D’Intino G, Giardino L, et al. (2004) Thyroid hormone administration enhances remyelination in chronic demyelinating inflammatory disease. Proc Natl Acad Sci USA 101(46): 16363-16368. 